\title{
EFFECT OF INTRALIGAMENTARY ANAESTHESIA IN REDUCING PAIN DURING PRIMARY MOLARS EXTRACTIONS UNDER GENERAL ANESTHESIA: A RANDOMIZED CLINICAL TRIAL
}

\author{
Laila Moustafa El Habashy* and Hisham Mohamed Gamal Eldine**
}

\begin{abstract}
Dentist anesthetists are always recommending the use of local anesthesia (LA) during tooth extractions to block any noxious stimulus and maintain the child vital signs with minimal interference.

Objective: To evaluate the effect of intraligamentry anaesthesia (ILA) in reducing child pain and maintaining the vital signs during mandibular primary molars extraction under general anaesthesia (GA).

Study design: A split-mouth controlled randomized clinical study was performed on 20 healthy children age ranging from 4-5 years indicated for complete oral rehabilitation under GA, with at least two bilateral mandibular primary molars indicated for extractions. A total of 40 primary molars were extracted, teeth were randomly assigned into two groups: Group A (extraction with ILA) and Group B (extraction without LA). Heart rate (HR) and mean arterial blood pressure (MABP) were used as parameters of pain, they were recorded before extraction (baseline data), and during the extraction procedure. The need of anesthetist intervention during extractions was also recorded.

Results: There was no statistically significant difference between the baseline vital parameters (HR, MABP) and during extraction using ILA ( $\mathrm{p}=0.195, \mathrm{p}=0.133$ respectively). There was a statistically significant increase in the vital parameters (HR, MABP) during extractions without LA compared to both the baseline and during extraction using ILA $(p=0.000 *)$. The need of anaesthetist intervention was statistically greater when extracting without LA than during extraction with $\operatorname{ILA}(\mathrm{p}=0.00026)$.
\end{abstract}

KEYWORDS: Primary molar, local anaesthesia, Intraligamentry anaesthesia, oral rehabilitation under GA

* Assistant Professor of Pediatric Dentistry, Pediatric Dentistry and dental Public Health Department, Faculty of Dentistry, Alexandria University.

** Lecturer anaesthesiology, Anaesthesia and Surgical Intensive Care Department, Faculty of Medicine, Alexandria University 


\section{INTRODUCTION}

GA is considered a method of advanced behaviour management used by dentists to allow for high quality dental treatments in children who otherwise cannot be managed on the dental chair. ${ }^{(1)}$ The use of LA in children undergoing complete oral rehabilitation under GA is a new concept to control pain and is recommended by the majority of anesthetist.(2) There is evidence that the use of LA can help reduce the amount of inhalational GA, produce a more hemodynamically stable patient, and controls pain in the immediate postoperative period. ${ }^{(3-5)}$ The properties of an ideal anaesthetic agent should provide: immobility; amnesia; sedation; analgesia, arousal blockade with a wide margin of safety, however, some anaesthetic agents do not provide profound amnesia. Alkire and Gorski, (6) proved that patients recovering from halothane and/or sevoflurane, were likely to pass through a phase where there is increased sensitivity to pain and increased tendency to remembering the experience, they concluded that the increase memory effect explains the intraoperative awareness when lighter anaesthesia phases were encountered. Therefore, most of the dentist anaesthetist recommends the use of LA during painful procedures especially tooth extraction under GA to reduce child pain and to maintain vital signs with minimal interference. ${ }^{(7)}$ Atan et al. ${ }^{(8)}$ and Sammons et al. ${ }^{(9)}$ showed a statistically significant decrease in pain following extractions if LA was added. Watts et al. ${ }^{(2)}$ found the heart rate and end-tidal carbon dioxide stayed stable for patients undergoing dental treatment under GA with supplemental LA, and were statistically lower than children without LA. In addition, patients with LA required less frequent anaesthesiologist intervention.

Inferior alveolar nerve block (IANB) is the most commonly used LA for mandibular molars extractions. This technique blocks the pain perception in all areas innervated with the mandibular nerve, as a result, patients encounter loss of sensitivity as well as total or partial loss of function of the facial muscles and tongue for the duration of the anaesthesia which may last up to two hours. ${ }^{(10)}$ Temporary hindering of patients' daily life with limited speech ability, eating, and most importantly, the risk of cheeks and lip biting are major drawback of this technique. Moreover, a rare but major complication of IANB anaesthesia is the injury of the mandibular nerve that could result in permanent impairment of the nerve function. ${ }^{(11,12)}$ In order to reduce these undesirable side effects first attempts for ILA was made at the beginning of the 20th century in order to anesthetize single tooth directly without influencing the nerve structures of the surrounding tissues. ${ }^{(13)}$ In 1920, Chompret, (14) published his experiences entitling his work "Anesthésie par injections intraligamenteuses". The anaesthesia was delivered to the tooth itself, anesthetizing the supporting tissue and the periodontal ligament, which are the fundamental of eliminating pain for any dental procedure including extractions. The onset of a successful anaesthesia occurred after about 40 seconds; and the average duration was about 30 minutes.

Although many studies have proven the effectiveness of ILA ${ }^{(15,16)}$ during different dental procedures, yet there are scarce documents in literature on the effectiveness of using ILA as a substitute to IANB during extractions in children undergoing complete oral rehabilitation under GA. Therefore, the objective of this study was to evaluate the effect of ILA in reducing child pain and maintaining the vital signs during mandibular primary molars extraction under GA.

The null hypothesis of this study was there will be no significant difference in the vital signs of the patients or the need of anaesthetist intervention during mandibular primary molars extraction under GA with or without the use of ILA. 


\section{METHODS}

\section{Ethical approval}

The ethical approval for this research protocol was obtained from the Research Ethics Committee at the Faculty of Dentistry, Alexandria University, Egypt, (Approval no. IORG0008839).

\section{Sample Size calculation ${ }^{(2,17,18)}$ :}

The minimal sample size was calculated based on a previous study aimed to estimate the use of LA in pediatric outpatient dental surgery. ${ }^{(2)}$ This study evaluated the physiologic stability, as determined by fluctuations in end-tidal carbon dioxide, heart rate, and respiratory rate, as well as subsequent anaesthetist intervention. Watts etal, in $2009^{(2)}$ concluded that patients who were not given LA were more liable to encounter vital sign fluctuation requiring anaesthetist intervention. Based on their results, adopting a power of $80 \%$ to detect a standardized effect size in heart rate fluctuation (primary outcome) of 0.812, and level of significance 95\% ( $\alpha=0.05)$, the minimum required sample size was found to be 20 teeth per group (number of groups=2) (Total sample size $=40$ teeth) ${ }^{(17)}$ is the minimum required sample size. Any withdrawal for any reason will be compensated by replacement to control for attrition (withdrawal) bias. ${ }^{(18)}$

\section{Sample selection}

One experienced pediatric dentist recruited the sample of this study by examining children attending the Pediatric Dentistry Clinics at the Faculty of Dentistry, Alexandria University. It included 20 children aged ranged from 4-5 years old with Frankl behaviour rating 1: Definitely negative, ${ }^{(19)}$ who needed complete oral rehabilitation under GA. Written informed consent was taken from all parents on the examination visit. Patients entering the study were otherwise healthy.

Study design: This study was a split-mouth, randomized controlled clinical trial.
Tooth inclusion criteria: Each child selected had at least bilateral mandibular primary molars that needed extractions, so that a primary molar on one side was extracted with ILA, and the contralateral was extracted without LA. Teeth were selected based upon the following clinical and radiographic criteria: clinically, teeth were included if they had non-restorable crowns with clinical signs and symptoms of extensive pulp degeneration, such as swelling, or fistula. Radiographically, the recruited teeth should have periapical or inter-radicular pathosis and/or pathological root resorption. ${ }^{(20)}$ Teeth showing any sign of mobility, root resorption more than one third of the root, ankyloses or root fracture were excluded from the study.

Of the 40 screened children, 20 met the aforementioned inclusion criteria. Exclusion was based on the refusal of parent/guardian to participate in the study ( 2 children), presence of tooth mobility (5 children), and pathological root resorption more than one third of the root (13 children).

Randomization and blindness: The allocation sequence was greated using a permuted block randomization technique, and the block size was variable. (21) Allocation sequence/code was concealed from the person assigning the participants to the intervention arms using sealed opaque envelopes. (22) Blinding could not be applied to the operators, anaesthesiologists, and recorders as data was based on objective readings from physiologic monitors.

Interventions: A total of 40 mandibular primary molars in 20 children were extracted during complete oral rehabilitation under GA. The same anaesthetic regimen was applied with all patients: Rapid mask induction was used with $8 \%$ sevoflurane in $4 \mathrm{~L} /$ minute of oxygen. Then, shortly after the patient lost consciousness, sevoflurane was changed to $4 \%$ in $4 \mathrm{~L} /$ minute of oxygen. An intravenous line was inserted. Cisatracurium was given in a dose of $0.15-0.2 \mathrm{mg} / \mathrm{kg}$ IV. Patient had nasal intubation; maintenance was achieved with isoflurane titrated to the desired effect. Boluses of intravenous 
propofol was given throughout the case, as indicated. After the induction, of the throat pack the initial vital signs including heart rate (HR) (beats per minute), and mean arterial blood pressure (MABP) were recorded.

In this study one experienced pediatric dentist performed all the extractions, in each patient, teeth were randomly assigned to either a study group or a control group, where one tooth was extracted after giving ILA (study) and the other tooth was extracted without LA (control). ILA administration was done as follow: pistol type syringe (Intraligamentry syringe (AM) ML 1.8 4962/2, Medesy ${ }^{\odot}$ Italy) was used, local anesthesetic agent mepivacaine hydrochloride, epinephrine free (Mepicaine 3\%, Alexandria Company for Pharmaceuticals and chemical industries, Alexandria, Egypt) was loaded into the syringe (Figure 1), extra short needle (Morita Dental Needles 30G Extra short M-NEEDLE30XS) was inserted at an angle of 30-40 degrees in respect to the long axis of the tooth and reached a sub-gingival depth of about 2 to $3 \mathrm{~mm}$ in the periodontal ligament. By pressing on the gun of the syringe a $0.2 \mathrm{ml}$ of the anaesthetic solution was pumped at each injection site, each root received two injections: mesial root (at the mesio-buccal and mesio-lingual line angle), Distal root (at the disto-buccal and disto-lingual line angles). The onset of a the anesthesia was after 40 seconds, and the average duration was 30 minutes. The dentist announced when each extraction procedure was going to be started. Using full crown mandibular pediatric forceps (Tooth Forceps Pediatric with Spring N.150 2600/150, Medesy ${ }^{\complement}$
Italy) extraction of primary molars was performed according to the American Academy of Pediatric Dentistry (AAPD) guidelines ${ }^{(23)}$ by applying slow continuous bucco/lingual force until expansion of the alveolar bone was achieved to accommodate the divergent roots of the primary molars reducing the risk of their fracture. Care was taken to support the mandible to protect the temporomandibular joints from injury. Finally, absorbable sutures (3/0, 18" Coated Vicryl Violet Braided Absorbable Suture with Reverse Cutting FS-2 Needle 3/8 circle, 19.0 mm. \#J393H, Ethicon Vicryl, USA) were taken to reduce the risk of postoperative bleeding and aspiration ${ }^{24)}$ (Figure 2).

The vital signs (HR and MABP) were recorded during extractions and the anaesthesiologist intervention was registered as simply "yes" or "no" for each extraction. The anaesthesiologist intervened when the following criteria were found: Patient movement in spite giving muscle relaxant with vital sign increased 20 percent above baseline. When intervention was needed, the patient received propofol in $10 \mathrm{mg}$ boluses. All patients received intravenous pain control 30 minutes before the end of the case, according to the Dental Surgery Center (DSC) standard pain management protocol of $1 \mathrm{mg} / \mathrm{kg}$ of Ketorolac (Toradol/Roche, Basel,Switzerland) to a maximum dose of $30 \mathrm{mg}$. ${ }^{(25)}$

For all patient enrolled in this study preventive orthodontic was done by constructing space maintainers appliances to maintain the space of the extracted primary molars to allow the proper eruption and alignment of the underlying permanent teeth. ${ }^{(26)}$

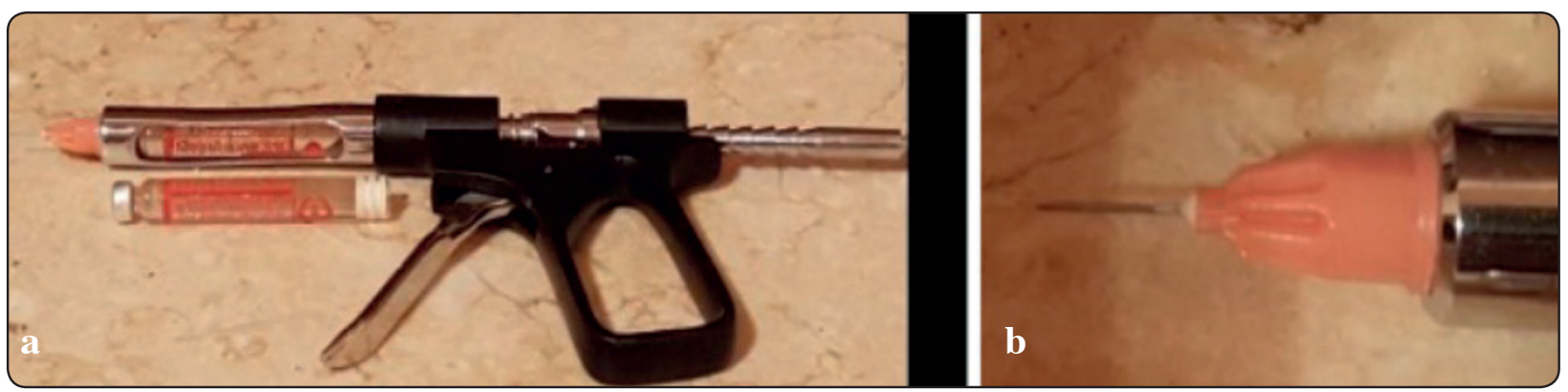

Fig. (1) a) photograph showing the PDL syringe, b) extra short needle 30G. 

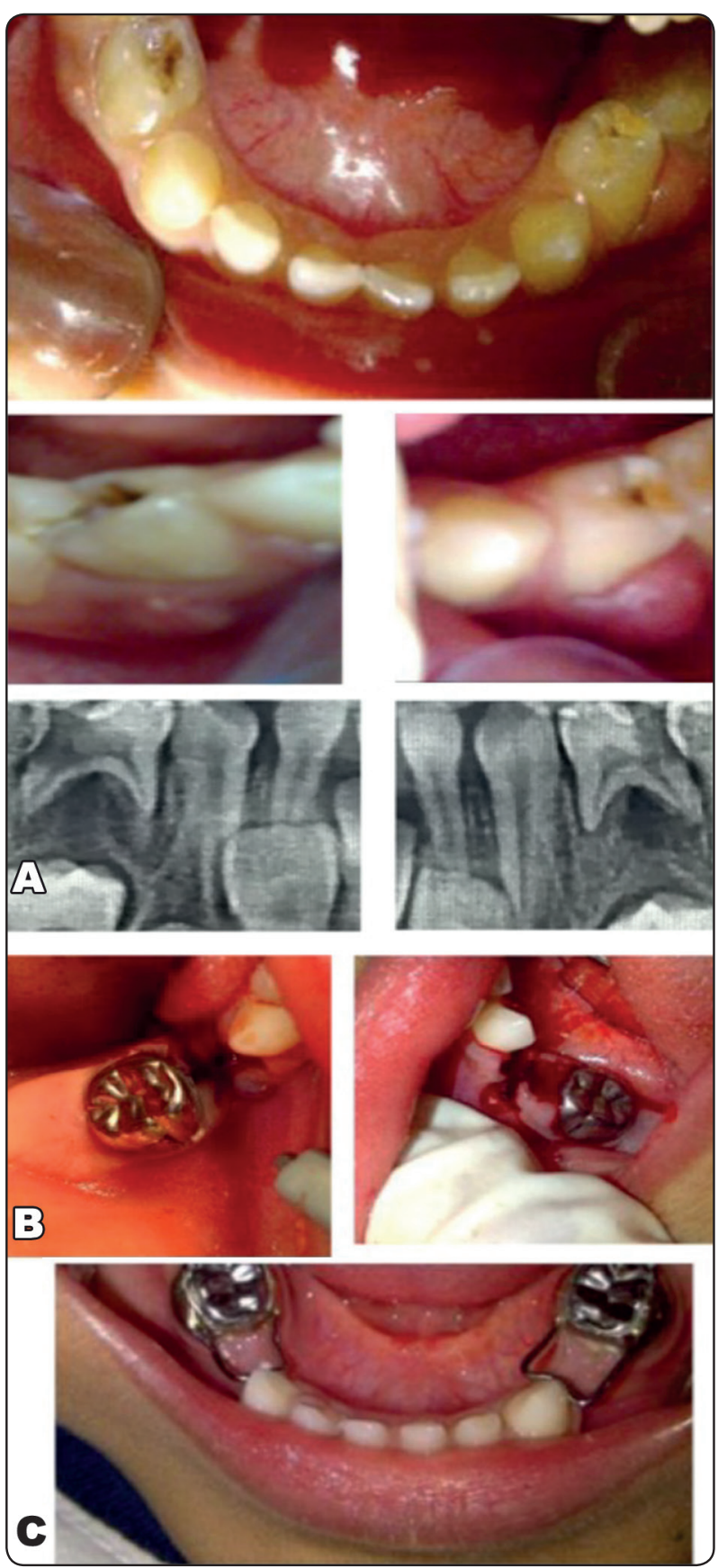

Fig. (2) Photographs showing a complete clinical case: a. preoperative picture showing bilateral clinical and radiographic abscessed lower first primary molars indicated for extractions, b. Extraction of both primary molars and suturing of the sockets (IL anesthesia given to the lower right first primary molar, no LA was given to the left molar).c.one month post-operative photographs showing 2 band and loop space maintainers.

\section{Statistical methodology ${ }^{(27,28)}$ :}

Data were collected and entered to the computer using SPSS (Statistical Package for Social Science) program for statistical analysis (ver 21). Data were entered as numerical or categorical, as appropriate. Kolmogorov-Smirnov test of normality revealed no significance in the distribution of the variables, so the parametric statistics was adopted. Data were described using minimum, maximum, mean, standard deviation and 95\% CI of the mean. Categorical variables were described using frequency and percentage. Comparisons were carried out between two studied dependent normally distributed variables using paired t-test. Z-test for independent proportions is used to compare two independent proportions. An alpha level was set to $5 \%$ with a significance level of $95 \%$. When calculating the sample size, a beta error accepted up to $20 \%$ with a power of study of $80 \%$.

\section{RESULTS}

A CONSORT diagram showing the study protocol is presented in Figure 3. (29)

Twenty patients (eight boys (40\%) and twelve girls $(60 \%)$ ) with a mean age of $4.70 \pm 0.50$ years participated in this study. Each child had one pair of their primary molars extracted under GA either with supplemental ILA (study group), or without LA (control group) (Table 1).

Table 2 shows the Vital signs parameter of pain; HR and MABP; before extraction (baseline), and during extractions either with ILA (study group) or without LA (control group). There was a statistical significant increase in the mean HR from the base line $(99.90 \pm 16.28)$ and during extraction with-

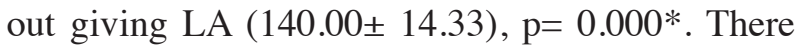
was no statistical significant difference between the mean HR at baseline and during extraction with ILA (102.15) $\mathrm{p}=0.195$. There was statistical significant difference between the mean HR during extraction with ILA and without LA $p=0.000 *$. 
There was a statistical significant increase in the MABP from the base line $(90.15 \pm 5.94)$ and during extraction without giving LA $(106.75 \pm 5.06)$ $\mathrm{p}=0.000^{*}$. There was no statistical significant difference between the MABP at baseline and during extraction with ILA $(91.38 \pm 5.84) \mathrm{p}=0.133$. There was statistical significant difference between the MABP during extraction with ILA and without LA, $\mathrm{p}=0.000^{*}$.

Regarding anaesthetist intervention: 10/20 patients needed anaesthetist intervention during extraction without LA (50\%) vs 0/20 (0\%) during extraction with ILA, this difference was statistically significant $(\mathrm{Z}=3.6515, \mathrm{p}=0.00026)$.

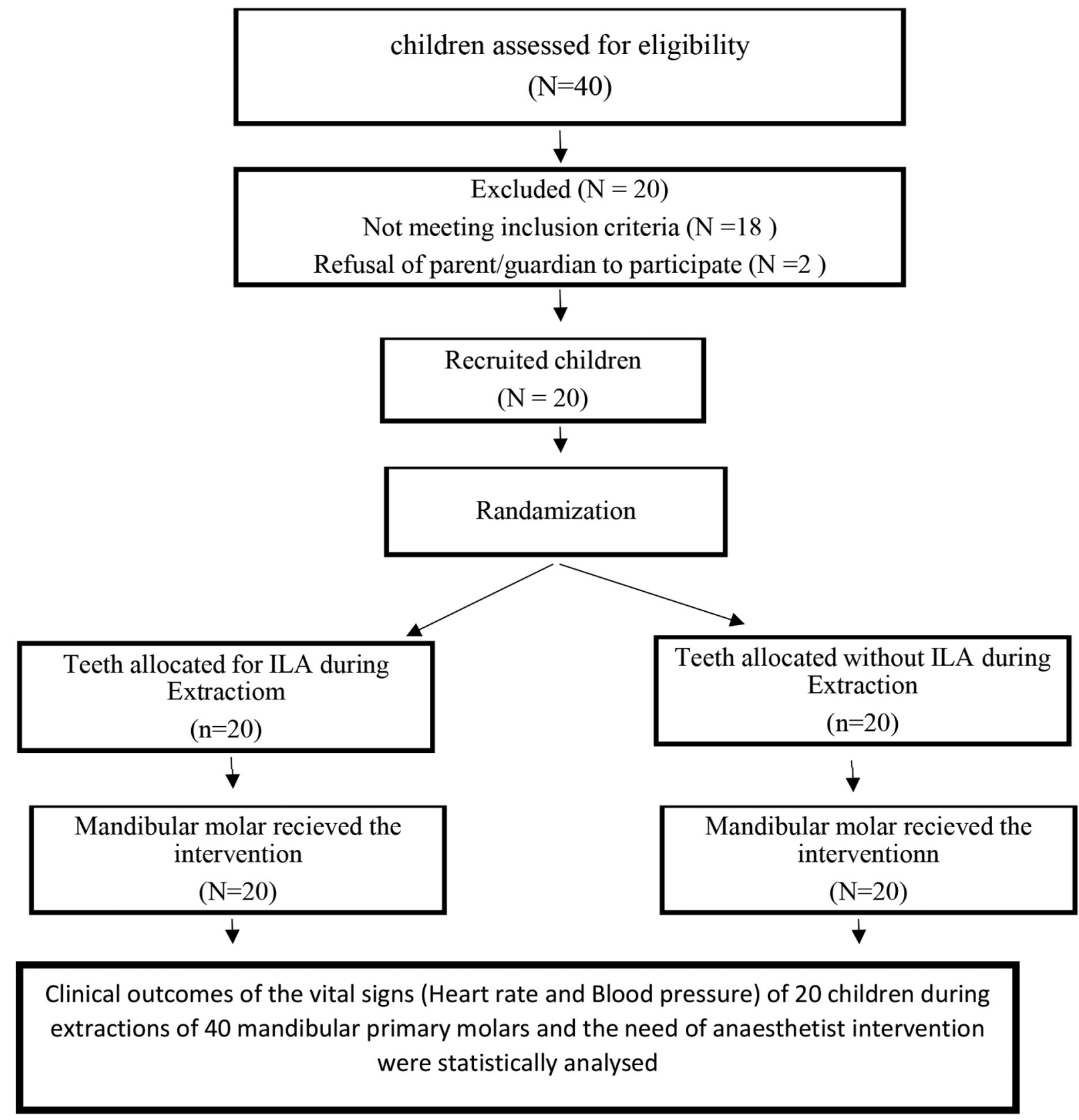

Fig. (3) A CONSORT diagram showing the study protocol. 
TABLE (1) Showing Demographic Distribution, Vital Signs records, and Patient's movement with Anaesthetist Intervention.

\begin{tabular}{|l|l|l|l|l|}
\hline case & age & sex & Mandibular molar extracted with ILA & Mandibular molar extracted without LA \\
\hline 1 & 4.2 & Girl & LLE & LRE \\
\hline 2 & 4.4 & Boy & LRE & LLE \\
\hline 3 & 5.1 & Girl & LRE & LLE \\
\hline 4 & 4 & Girl & LLD & LRD \\
\hline 5 & 4.3 & Boy & LRD & LLD \\
\hline 6 & 5.2 & Girl & LLE & LRE \\
\hline 7 & 5.2 & Girl & LRD & LLD \\
\hline 8 & 5.1 & Girl & LRE & LLE \\
\hline 9 & 5 & Girl & LRD & LLD \\
\hline 10 & 4.1 & Boy & LLD & LRD \\
\hline 11 & 4.5 & Girl & LLD & LRD \\
\hline 12 & 4.3 & Boy & LRD & LLD \\
\hline 13 & 5.3 & Girl & LRD & LLD \\
\hline 14 & 5.3 & Boy & LLD & LRD \\
\hline 15 & 5.2 & Boy & LRE & LLE \\
\hline 16 & 4.6 & Girl & LRD & LLD \\
\hline 17 & 4.2 & Boy & LRE & LLE \\
\hline 18 & 4.5 & Girl & LRD & LLD \\
\hline 19 & 5 & Boy & LLE & LRE \\
\hline 20 & 4.1 & Girl & LLE & LRE \\
\hline
\end{tabular}

TABLE (2) Showing the Vital signs parameter of pain (HR and MABP) before extraction (baseline), and during extractions in both groups.

\begin{tabular}{|c|c|c|c|}
\hline & Baseline & during extraction with ILA & during extraction without PDL \\
\hline \multicolumn{4}{|l|}{ HR (beats/min) } \\
\hline$-n$ & 20 & 20 & 20 \\
\hline - Min-Max & $75.00-130.00$ & $80.00-132.00$ & $120.00-160.00$ \\
\hline- Mean \pm SD & $99.90 \pm 16.28$ & $102.15 \pm 14.03$ & $140.00 \pm 14.33$ \\
\hline$-95 \% \mathrm{Cl}$ for mean & $92.28-107.51$ & $95-58-108.72$ & $133.29-146.70$ \\
\hline \multirow{4}{*}{ Paired sample $t$ test } & & $\mathrm{t}=1.342$ & $t=21.902$ \\
\hline & & $p=0.195 \mathrm{NS}$ & $p=0.000^{*}$ \\
\hline & & \multirow{2}{*}{\multicolumn{2}{|c|}{$\begin{array}{l}\mathrm{t}=15.581 \\
p=0.000 *\end{array}$}} \\
\hline & & & \\
\hline \multicolumn{4}{|l|}{ MABP $(\mathrm{mmHg})$} \\
\hline$-n$ & 20 & 20 & 20 \\
\hline - Min-Max & $81.67-100.00$ & $83.33-102.67$ & $96.67-117.67$ \\
\hline - Mean \pm SD & $90.15 \pm 5.94$ & $91.38 \pm 5.84$ & $106.75 \pm 5.06$ \\
\hline$-95 \% \mathrm{Cl}$ for mean & $87.37-92.93$ & $88.65-94.11$ & $104.38-109.12$ \\
\hline \multirow{4}{*}{ Paired sample $t$ test } & & $\mathrm{t}=1.571$ & $t=23.992$ \\
\hline & & $p=0.133 \mathrm{NS}$ & $p=0.000^{*}$ \\
\hline & & \multicolumn{2}{|l|}{$t=17.517$} \\
\hline & & \multicolumn{2}{|l|}{$p=0.000 *$} \\
\hline
\end{tabular}




\section{DISCUSSION}

The use of LA with GA has been advocated to improve physiological parameters during GA.

(2) Most of the anesthetists are recommending the use of LA in children undergoing complete oral rehabilitation under GA. In 2014 a survey study (7) listed the benefits of the use of LA as very important factors among 28 anesthetists as follows: maintenance of the vital signs and decreased depth of GA (92.9\%), smooth recovery (82.1\%), preventing the provoke of deep pain stimulus $(60.7 \%)$, and fast recovery $(57.1 \%)$.

Inferior alveolar nerve block (IANB) is the most common technique used to anesthetize mandibular molars, but the risk of cheeks and lip biting is one of its major drawback especially in children, Townsend JA, et al. ${ }^{(7)}$ found that members of the AAPD and American Dental association (ADA) who did not prefer to give LA in children undergoing complete oral rehabilitation under GA was mainly due to their concern of postoperative cheek and lip biting which they addressed as a major point of consideration when dealing with kids. Other members who preferred the use of LA mainly recommended its use during permanent or primary teeth extractions.

As ILA overcomes the limitation of IANB regarding cheek and lip biting, therefore this study aimed to evaluate the effect of ILA in reducing child pain and maintaining the vital signs during mandibular primary molars extraction under GA.

This study was a split-mouth design to exclude any individual variation in the vital signs, pain threshold, pain perceptions, and any other variations that might affect the outcome of the study. The children enrolled were between 4-5 years old with only one-year range span as it is well known that different age group in a growing child has different HR and MABP. ${ }^{(30)}$

In the present study the HR and the MABP were the primary vital signs that represented pain, this was supported by other studies in literature. (2) It is well known that the relation between pain, tachycardia and hypertension is a fundamental in internal medicine. Physiologically, acute pain stimulus evokes a stress response resulting in increased blood pressure, heart rate, pupil diameter, and plasma cortisol levels. ${ }^{(31)}$

In this study the anaesthetist intervened when the patient showed movement with an increase in the vital sign 20 percent above baseline, in such case the patient received propofol in $10 \mathrm{mg}$ boluses as the medication has a potent effect in suppressing awareness than nitrous oxide and opioids. ${ }^{(32)}$

The result of the present study showed a significant increase in HR and MABP from the baseline parameters and during extractions without LA indicating pain. On the other hand, there was no statistically significant difference between the $\mathrm{HR}$ and MABP at the baseline and during extraction using ILA indicating the effectiveness of the ILA in anesthetizing the tooth and eliminating pain. Furthermore, the vital signs readings were also significantly higher during extraction without LA than during extraction with ILA, and a significant number of children (50\%) undergoing extraction without LA (compared to none with ILA) showed movements and needed the anaesthetist intervention. In such cases propofol was given resulting in more time for patient recovery. These findings were in accordance with Watts et al. (2) who examined the physiologic effects of different dental procedures (rubber dam clamp placement, pulpotomy, cementation of a stainless-steel crowns, and extraction) on children undergoing GA; and the correlation between LA administration and therapeutic intervention by the anaesthetist. They concluded that children who were not given LA were more liable to encounter vital sign fluctuation especially during extractions that required anaesthetist intervention.

In this study the effectiveness of the ILA in anaesthetizing the tooth and eliminating pain was in 
agreement with Tekin U, et al. ${ }^{(33)}$ who compared in a randomized split moth clinical trial the effect of IANB and ILA on child discomfort during the LA admiration and during mandibular first molar extraction on the dental chair. Their study included 29 healthy children aged 8 to 9 years, HR values were recorded during the injection, and extraction periods, and the Pain perceptions were evaluated with sound, eye and motor (SEM) scale. Their results showed that although the HR was higher in IANB compared to ILA during both injection and extraction procedures but the differences were not statistically significant, while a significant higher mean in the SEM score for IANB group was recorded in comparison to ILA during both injection and extraction periods. On the other hand, the results of the present study were inconsistent with, Rayalat et al, ${ }^{(34)}$ who found that ILA was significantly less effective in eliminating pain during extraction of maxillary molars compared to infiltration anaesthesia, and the need of additional injections of LA to attain the desire painless extractions was necessary. Furthermore, in another study comparing the efficacy of Articaine 4\% to lidocaine 2\% when used as ILA during extractions of mandibular primary molars in sixty children, aged ranged from 6 to 10 years, it was found that using lidocaine ILA was effective in only $30 \%$ of the cases and the administration of IANB was needed during the extraction procedure. ${ }^{(35)}$ Our explanation to the different outcomes between our study and the aforementioned studies is attributed to the conjunction of ILA to the GA, it might be assumed that when the patient was under the effect of GA, the ILA was effective to block the painful stimulus of extractions while this technique might not be effective when the patient was fully awake on the dental chair.

Finally, it has to be noted that since the anaesthesia protocol of this study recommended giving the children propofol muscle relaxant, therefore, the respiratory rate could not be considered as a reliable parameter of pain which was considered as a limitation of this study.
The null-hypothesis of this study was rejected as there was a statistically significant difference between the clinical outcomes when using ILA during extraction of primary molars under GA and when extraction was done without LA.

\section{CONCLUSION}

Based on the results of the present study, it may be concluded that the need of LA during extraction under GA is mandatory to maintain patients' vital signs and decrease depth of GA and the need of anaesthetist intervention resulting in fast and smooth recovery. Intraligamentry anaesthesia has proved to be an effective technique in eliminating pain and maintaining the vital signs of the patients during extractions of primary mandibular molars under GA.

\section{RECOMMENDATION}

Intraligamentry anaesthesia should be an alternative technique to IANB during extractions of mandibular teeth under GA to eliminate the risk of cheek and lip biting postoperatively, which is a major point of consideration especially when dealing with kids.

Funding: This research received no funding.

Conflict of interest: Authors declare to conflict of interest

\section{REFERENCES}

1. American Academy of Pediatric Dentistry. Guideline on behavior guidance for the pediatric dental patient. Pediatr Dent 2011;36:179-91.

2. Watts AK, Thikkurissy S, Smiley M, McTigue DJ, Smith $\mathrm{T}$. Local anesthesia affects physiologic parameters and reduces anesthesiologist intervention in children undergoing general anesthesia for dental rehabilitation. Pediatr Dent 2009;31(5):414-9.

3. Shandling B, Steward DJ. Regional analgesia for postoperative pain in pediatric outpatient surgery. J Pediatr Surg 1980;15(4):477-80.

4. Dahl V, Gierløff C, Omland E, Raeder JC. Spinal, epidural or propofol anaesthesia for out-patient knee arthroscopy? Acta Anaesthesiol Scand 1997;41(10):1341-5. 
5. Noble DW, Raab GM, MacLean D, MacLachlan D. Prilocaine infiltration as postoperative analgesia for children having dental extractions under general anesthesia. Reg Anesth 1994;19(2):126-31.

6. Alkire MT, Gorski LA. Relative amnesic potency of five inhalational anesthetics follows the Meyer-Overton rule. Anesthesiology 2004;101(2):417-29.

7. Townsend JA, Hagan JL, Smiley M. Use of local anesthesia during dental rehabilitation with general anesthesia: a survey of dentist anesthesiologists. Anesth Prog 2014;61(1):11-7.

8. Atan S, Ashley P, Gilthorpe M, Scheer B, Mason C, Roberts G. Morbidity following dental treatment of children under intubation general anaesthesia in a day-stay unit. Int J Paediatr Dent 2004;14(1):9-16.

9. Sammons HM, Unsworth V, Gray C, Choonara I, Cherrill J, Quirke W. Randomized controlled trial of the intraligamental use of a local anaesthetic (lignocaine 2\%) versus controls in paediatric tooth extraction. Int J Paediatr Dent 2007;17(4):297-303.

10. Sambrook PJ, Goss AN. Severe adverse reactions to dental local anaesthetics: prolonged mandibular and lingual nerve anaesthesia. Aust Dent J 2011;56(2):154-9.

11. Alhassani AA, AlGhamdi AS. Inferior alveolar nerve injury in implant dentistry: diagnosis, causes, prevention, and management. J Oral Implantol 2010;36(5):401-7.

12. Needleman HL. Local anesthesia during dental rehabs, "To use, or not to use: that (still) is the question.”. Pediatr Dent 2010;32(1):7.

13. Meechan JG. Intraligamentary anaesthesia. J Dent 1992; 20(6):325-32

14. Chompret L. Anesthésie par injections intraligamenteuses. Rev Stomatol Chir Maxillofac 1920;6:309-12.

15. Kämmerer P, Adubae A, Buttchereit I, Thiem D, Daubländer M, Frerich B. Prospective clinical study comparing intraligamentary anesthesia and inferior alveolar nerve block for extraction of posterior mandibular teeth. Clin Oral Investig 2018;22(3):1469-75.

16. Thiem DGE, Schnaith F, Van Aken CME, Köntges A, Kumar VV, Al-Nawas B, et al. Extraction of mandibular premolars and molars: comparison between local infiltra- tion via pressure syringe and inferior alveolar nerve block anesthesia. Clin Oral Investig 2018;22(3):1523-30.

17. Charan J, Biswas T. How to calculate sample size for different study designs in medical research? Indian J Psychol Med 2013;35(2):121-6.

18. Faul F, Erdfelder E, Lang A-G, Buchner A. G* Power 3: A flexible statistical power analysis program for the social, behavioral, and biomedical sciences. Behav Res Methods 2007;39(2):175-91.

19. Frankl S. Should the parent remain with the child in the dental operatory? J Dent Child 1962;29:150-63.

20. Dhar V, Marghalani AA, Crystal YO, Kumar A, Ritwik $\mathrm{P}$, Tulunoglu $\mathrm{O}$, et al. Use of vital pulp therapies in primary teeth with deep caries lesions. Pediatr Dent 2017; 39(5):146E-59E.

21. Schulz KF, Grimes DA. Allocation concealment in randomised trials: defending against deciphering. The Lancet 2002;359(9306):614-8.

22. Schulz KF, Grimes DA. Generation of allocation sequences in randomised trials: chance, not choice. The Lancet 2002;359(9305):515-9.

23. American Academy of Pediatric Dentistry. Guideline on on pediatric Oral Surgery. J Pediatric Dentistry 2013;35(5):238-45

24. Hu YH, Tsai A, Ou-Yang LW, Chuang LC, Chang PC. Postoperative dental morbidity in children following dental treatment under general anesthesia. BMC Oral Health 2018;18(1):84

25. Abbas SM, Kamal RS, Afshan G. Effect of ketorolac on postoperative pain relief in dental extraction cases-a comparative study with pethidine. J Pak Med Assoc 2004;54(6):319-22.

26. Dean JA. Managing the developing occlusion. McDonald and Avery's Dentistry for the Child and Adolescent. 10th ed. Maryland Heights, MO.: Mosby Elsevier; 2016. p. 415-78.

27. IBM Corp. (Released 2012). IBM SPSS Statistics for Windows, Version 21.0. Armonk, NY: IBM Corp; 2012.

28. Karanicolas PJ, Farrokhyar F, Bhandari M. Blinding: who, what, when, why, how? Can J Surg 2010;53(5):345.

29. Schulz KF, Altman DG, Moher D, Consort Group. CONSORT 2010 statement: updated guidelines for reporting parallel group randomised trials. Int J Surg 2011; 9(8):672-7. 
30. Coulthard MG. Single blood pressure chart for children up to 13 years to improve the recognition of hypertension based on existing normative data. Arch Dis Child 2020;105(8):778-83.

31. Rathmell JP, Fields HL. Pain: pathophysiology and management. In: Longo D, Fauci A, Kasper D, Hauser S, Jameson J, Loscalzo J, editors. Harrison's Principles of Internal Medicine, 18th Edition. New York, NY: Mcgrawhill; 2011.p. 93-101.

32. Dwyer R, Bennett HL, Eger EI, 2nd, Heilbron D. Effects of isoflurane and nitrous oxide in subanesthetic concentrations on memory and responsiveness in volunteers. Anesthesiology 1992;77(5):888-98.
33. Tekin U, Ersin N, Oncag O, Bent B, Menderes M, Kocanali B. Comparison of inferior alveolar nerve block and intraligamentary anesthesia on the discomfort of children. JIDMR 2012;5(3):143-8.

34. Ryalat ST, Al-Shayyab MH, Amin W, AlRyalat SA, AlRyalat N, Sawair F. Efficacy of intraligamentary anesthesia in maxillary first molar extraction. J Pain Res 2018; 11:1829-33.

35. Sharan S, Goswami M, Kaul R, Rahman B, Farooq S. Comparative evaluation of effectiveness of intraligamentary injection technique using articaine and lidocaine for extraction of primary mandibular posterior teeth. Int J Pedod Rehabil 2018;3(2):62-6. 